\title{
BMJ Open Frameworks, measures, and interventions for HIV-related internalised stigma and stigma in healthcare and laws and policies: systematic review protocol
}

\author{
Susanne Hempel (10 , ${ }^{1}$ Laura Ferguson, ${ }^{2}$ Maria Bolshakova, ${ }^{1}$ Sachi Yagyu, ${ }^{1}$ \\ Ning Fu, ${ }^{3}$ Aneesa Motala, ${ }^{1}$ Sofia Gruskin ${ }^{2}$
}

To cite: Hempel S,

Ferguson L, Bolshakova M, et al. Frameworks, measures, and interventions for HIVrelated internalised stigma and stigma in healthcare and laws and policies: systematic review protocol. BMJ Open 2021;11:e053608. doi:10.1136/ bmjopen-2021-053608

- Prepublication history and additional supplemental material for this paper are available online. To view these files, please visit the journal online (http://dx.doi.org/10.1136/ bmjopen-2021-053608)

Received 24 May 2021 Accepted 30 September 2021

A Check for updates

(C) Author(s) (or their employer(s)) 2021. Re-use permitted under CC BY. Published by BMJ.

${ }^{1}$ Southern California Evidence Review Center, University of Southern California, Los Angeles, California, USA

${ }^{2}$ Institute on Inequalities in Global Health, University of Southern California, Los Angeles, California, USA

${ }^{3}$ Department of Economics, Shanghai University of Finance and Economics, Shanghai, China

Correspondence to

Dr Susanne Hempel;

susanne.hempel@med.usc.edu

\section{ABSTRACT}

Introduction There is strong global commitment to eliminate HIV-related stigma. Wide variation exists in frameworks and measures, and many strategies to prevent, reduce or mitigate stigma have been proposed but critical factors determining success or failure remain elusive.

Methods and analysis Building on existing knowledge syntheses, we designed a systematic review to identify frameworks, measures and intervention evaluations aiming to address internalised stigma, stigma and discrimination in healthcare, and stigma and discrimination at the legal or policy level. The review addresses four key questions (KQ): KQ1: Which conceptual frameworks have been proposed to assess internal stigma, stigma and discrimination experienced in healthcare settings, and stigma and discrimination entrenched in national laws and policies? KQ2: Which measures of stigma have been proposed and what are their descriptive properties? KQ3: Which interventions have been evaluated that aimed to reduce these types of stigma and discrimination or mitigate their adverse effects and what are the effectiveness and unintended consequences? KQ4: What common 'critical factors for success or failure' can be identified across interventions that have been evaluated? We will search PubMed, PsycINF0, Web of Science, Universal Human Rights Index, HeinOnline, PAIS, HIV Legal Network, CDSR, Campbell Collaboration, PROSPERO and Open Science Framework. Critical appraisal will assess the source, processes and consensus finding for frameworks; COnsensus-based Standards for the selection of health Measurement Instruments criteria for measures; and risk of bias for interventions. Quality of evidence grading will apply . A gap analysis will provide targeted recommendations for future research. We will establish a compendium of frameworks, a comprehensive catalogue of available measures, and a synthesis of intervention characteristics to advance the science of HIV-related stigma. PROSPERO registration number CRD42021249348.

\section{INTRODUCTION}

Stigma is derived from a Greek word meaning a mark or stain. Much work around HIVrelated stigma uses as its starting point Goffman's 1963 definition as 'an attribute that is deeply discrediting'. ${ }^{1}$ This has been furthered by Hatzenbuehler et al who, bringing in the
Strengths and limitations of this study

- Extensive literature searches will summarise HIV stigma evidence.

- This review will establish a comprehensive compendium of frameworks, a user-friendly catalogue of existing measures, and a clear synthesis of the effects of interventions.

- The review is limited to internalised stigma, stigma and discrimination in healthcare, and stigma and discrimination at the legal or policy level.

idea of the power dynamics that allow the distancing or othering so often associated with stigma, define stigma as 'the co-occurrence of labelling, stereotyping, separation, status loss and discrimination in a context in which power is exercised'. ${ }^{2}$ Relf $e t$ al define stigma as 'a social phenomenon that occurs when a person is evaluated as having an undesirable trait, attribute or behaviour and is subsequently deemed imperfect by societal standards'. ${ }^{3}$ Each of these definitions brings in something slightly different and many of the core concepts are neatly summed up by Parker and Aggleton who note that 'stigma functions at the intersection of culture, power and difference'. ${ }^{4}$ Stigma can be described as a dynamic process of devaluation that significantly discredits an individual in the eyes of others, such as when certain attributes are seized on within particular cultures or settings and defined as discreditable or unworthy.

The term stigma is often used in the literature to encompass both stigma and discrimination even as these are conceptually distinct. While stigma refers to an attitude or belief, discrimination is the behaviour or action that results from those attitudes or beliefs. Hence, when stigma is acted on, the result can be discrimination. Discrimination may refer to any form of arbitrary distinction, 
exclusion or restriction affecting a person, usually (but not only) because of an inherent personal characteristic or perceived membership of a particular group.

HIV-related stigma has been defined by the Joint United Nations Programme on HIV/AIDS (UNAIDS) as negative beliefs, feelings and attitudes towards people living with HIV, groups associated with people living with HIV (eg, families of people living with HIV) and other key populations at higher risk of HIV infection, such as people who inject drugs, sex workers, men who have sex with men and transgender people. ${ }^{5}$ Although there is no universal consensus as to their categorisation, and many perceive these to be along a continuum, different domains have been identified within HIV-related stigma, including internalised, anticipated, perceived, enacted, externalised and structural stigma. Different types of stigmas can be experienced and assessed both alone and in combination, each of which is experienced differently and therefore must be addressed differently. Discrimination, as defined under international human rights law, is any distinction, exclusion or restriction based indirectly or directly on grounds prohibited under international law, which has the effect or intent of nullifying the recognition, enjoyment or exercise on an equal basis of others of all human rights and fundamental freedoms, in the political, economic, social, cultural, civil or any other field. ${ }^{6}$ In the case of HIV, this can be discrimination based on a person's real or perceived HIV-positive status, irrespective of whether or not there is any justification for these measures. ${ }^{7}$ It can rise to the level of a human rights violation. HIV-related discrimination is therefore any distinction, exclusion or restriction (sometimes referred to as acts or omissions) based indirectly or directly on a person's real or perceived HIV status. ${ }^{8}$

There is strong global commitment to eliminate HIVrelated stigma, starting with global political commitments and reflected in global and national strategies as well as the multitude of organisations and collaborations working to address stigma. ${ }^{9}{ }^{10}$ Yet, learning across interventions designed to mitigate against the experience and harmful impacts of stigma is limited by the multitude of evaluation frameworks and measures in use to assess the different dimensions of stigma. For example, a recent review of interventions to address self-stigma did not include a formal meta-analysis due to the heterogeneity of measures used, with eight different scales used across 20 studies. $^{7}$ A 2015 UNAIDS report documented over 60 tools to assess and/or address stigma and discrimination within healthcare settings. ${ }^{11}$ Assessment of stigma and discrimination entrenched in laws and policies also takes multiple forms including the People Living with HIV Stigma Index, Module 6 of the Demographic and Health Survey, the Integrated Bio-Behavioural Survey, legal environment assessments, the Global Fund baseline assessment methodologies, and the National Commitments and Policy Instrument of the Global AIDS Monitoring process.
Understanding the state of the research in relation to measurement of self-stigma, in accessing health services, and in laws and policies, is needed to help inform future efforts, at all levels, to better address stigma and support people's health and well-being. This review will systematically identify and assess frameworks, measures and interventions of HIV-related individual internalised stigma; both stigma and discrimination within healthcare settings; and stigma and discrimination at the legal and policy level. The intersectionality of HIV-related stigma with other forms of stigma such as stigma relating to 'key' and 'vulnerable' populations that are disproportionately affected by HIV is critical. The review will focus on HIVrelated stigma itself, acknowledging as possible where intersectionality is addressed and, specifically, if there appear to be particular gaps in attention to stigma with respect to specific population groups.

\section{Review questions}

The systematic review will be guided by four key questions.

Key question 1

Which conceptual frameworks have been proposed to assess internal stigma, stigma and discrimination experienced in healthcare settings, and stigma and discrimination entrenched in national laws and policies?

\section{Key question 2}

Which measures (eg, assessment scales) of these different types of stigma and discrimination have been proposed and what are their descriptive properties?

\section{Key question 3}

Which interventions have been evaluated that aimed to reduce these types of stigma and discrimination or mitigate their adverse effects and what are the effectiveness and unintended consequences of the interventions?

\section{Key question 4}

What common 'critical factors for success or failure' can be identified across the interventions that have been evaluated that might inform future interventions?

\section{METHODS AND ANALYSIS}

The reporting of the protocol and the review follow the Preferred Reporting Items for Systematic Reviews and Meta-Analyses guidelines. The systematic review is part of a larger project undertaken by the International AIDS Society (IAS). It is accompanied by four national initiatives to explore stigma and discrimination in the local contexts of Kenya, Malawi, South Africa and Zambia through key informant interviews and grey literature searches. The project started in November 2020 and is planned to be completed by March 2022.

The systematic review will follow a transparent and rigorous procedure to minimise review selection and reporting bias. We will search multiple disciplinary and interdisciplinary sources to ensure all relevant studies 
are captured. Citations and full text publications will be screened by independent literature reviewers to reduce reviewer errors and bias. Eligibility decisions, including reasons for exclusions, will be tracked in citation management software. Data abstraction and critical appraisal will be conducted in online software designed for systematic reviews using detailed, pilot-tested forms with clear reviewer instructions to avoid ambiguity and ensure replicability of coding decisions. The collected data will be accessible in a review data repository. ${ }^{12}$

\section{Context}

Our systematic review will be embedded in the context of existing research syntheses. To date, a substantial number of systematic reviews has been published that address different aspects of HIV-related stigma and discrimination. These include associations of stigma (covariates, causes or effects),${ }^{13-40}$ access to care,${ }^{41-66}$ HIV testing,${ }^{67-91}$ country-specific explorations of stigma or discrimination, ${ }^{92-109}$ the role of stigma and discrimination in treatment adherence, ${ }^{110-127}$ HIV experiences of people living with HIV or their care givers, ${ }^{128-141}$ stigma/discrimination reduction in the community, ${ }^{142-153}$ HIV disclosure considerations, ${ }^{154-162}$ stigma/discrimination in healthcare, ${ }^{163-170}$ rights and regulations, ${ }^{171-177}$ intersectionality, ${ }^{178-183}$ measuring stigma, ${ }^{184-189}$ HIV prevention, ${ }^{190-193}$ stigma reduction in low income countries, ${ }^{194-197}$ self-stigma ${ }^{7198} 199$ and other, unique topics. ${ }^{200} 201$ We build on these reviews which outline existing research and point to persistent knowledge gaps. The reviews will be instrumental for reference-mining to ensure that all relevant material has been considered.

We will identify and categorise existing systematic reviews to explore the research field further. Systematic reviews will be identified through PubMed (biomedical literature) using the systematic review filter, through PsycINFO (psychosocial literature) and Web of science (general science literature including legal and policy analysis), as well as through the Cochrane Database of Systematic Reviews (focus on health) and the Campbell Collaboration (focus on social sciences). Furthermore, we will search the review registries PROSPERO and Open Science Framework to ensure that all relevant registered systematic reviews have been identified.

\section{Analytical framework}

Given that there is no agreed nomenclature in this interdisciplinary field, we established working definitions of the concepts 'stigma,' 'internalised stigma' and 'discrimination' for the purpose of this systematic review:

- Stigma refers to beliefs and/or attitudes about HIV.

- Discrimination refers to the behaviours that result from attitudes or beliefs about HIV.

- Internalised stigma (self-stigma) refers to a person living with HIV internalising negative attitudes associated with HIV and accepting these as applicable to themselves.

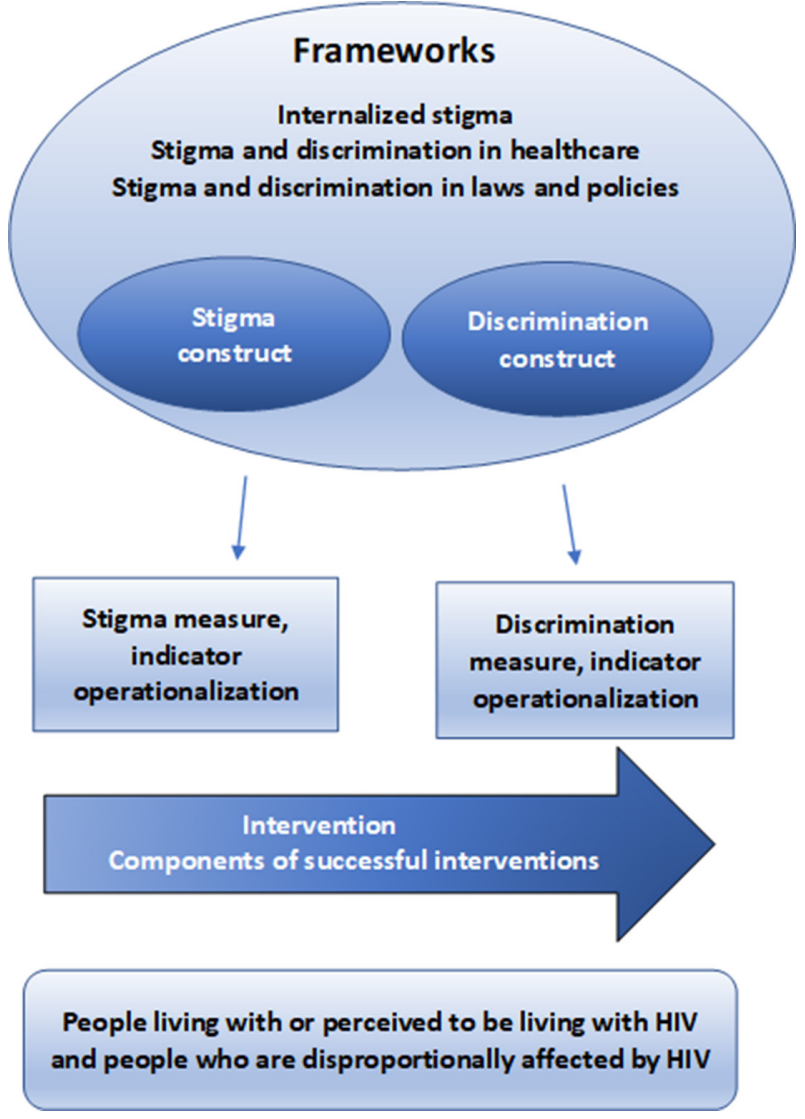

Figure 1 Analytical framework.

- Stigma and discrimination in healthcare refers to negative beliefs and behaviours based on perceived or actual HIV status experienced in healthcare delivery settings.

- Stigma in laws and policies refers to distinctions, exclusion or restriction based on perceived HIV status or membership of a group that is vulnerable to HIV.

The analytical framework depicted in figure 1 maps the review's four Key Questions and outlines the review's content, that is, frameworks, measures and intervention evaluations, targeted in this systematic review.

The figure includes existing conceptual models for HIVrelated stigma, including frameworks such as the global HIV stigma reduction framework, ${ }^{202} 203$ and logic models addressing stigma and discrimination, potential causes or effects (key question 1). The frameworks comprise of the underlying construct of stigma and/or discrimination and their components. The analytical framework shows also the measures, indicators or operationalisations of the constructs stigma and discrimination that are used to measure and quantify stigma and discrimination (key question 2). The arrow represents interventions aiming to prevent, reduce, or mitigate stigma and discrimination (key question 3), as well as the 'critical success factors' of tested interventions (key question 4).

The systematic review is focused on people with living or perceived to be living with HIV and people who are disproportionately affected by HIV. For the purposes of 
this review and based on the UNAIDS definition, the latter group includes gay men and other men who have sex with men, sex workers, transgender people, people who inject drugs and prisoners and other incarcerated people. ${ }^{204}$

\section{Search strategy}

To identify primary research studies, we will search the health-specific research database PubMed, in particular to identify research on stigma experienced in healthcare settings. We will search PsycINFO to identify psychological and social research on stigma and use the general scientific research database Web of Science, in particular to identify legal and policy analyses on stigma and discrimination. We will identify government and nongovernmental organisation reports indexed in the Universal Human Rights Index, HeinOnline, PAIS and HIV Legal Network.

Additional grey literature searches will target organisations relevant to key populations affected by HIV and funders of stigma research. Specifically, we will search the websites of the IAS, UNAIDS, United Nations Development Programme, STRIVE, Health Policy Plus and Sage (resource-sharing community for Canadian HIV and hepatitis $\mathrm{C}$ service providers).

Targeted search strategies for each key question will combine free-text search terms with controlled vocabulary of the individual databases. Draft search strategies are shown in online supplemental appendix 1 . The searches will be designed, executed and documented by an Evidence-based Practice Centre librarian. The searches outside of research databases will be instrumental in identifying conceptual models to assess internal stigma and stigma entrenched in national laws and policies. The multiple sources and interdisciplinary approach aim to reduce selection bias being introduced in the literature review.

\section{Eligibility criteria}

We use a participant, independent variable, comparator or study design, outcome/measure, timing and setting framework to structure the eligibility criteria:

\section{Participants}

People living with or perceived to be living with HIV and people from groups who are disproportionately affected by HIV infection. We will exclude studies of mixed populations and other participant targets unless the study provides HIV-relevant subgroup analyses.

\section{Independent variable}

Frameworks will include models outlining and relating to multiple components of stigma and/or discrimination, including conceptual frameworks, logic models, taxonomies and analytic models for assessment, prevention, reduction or mitigation of stigma. Measures will include self and peer report measures used for formal assessment of stigma and discrimination. Studies using a published measure will be included if the psychometric properties of the measure are a central focus of the study (eg, evaluating the predictive validity of an existing measure). Eligible intervention evaluations may test strategies and policies aimed at preventing, reducing or mitigating HIV-related stigma and discrimination. Intervention evaluations aimed at self-stigma, stigma and discrimination in healthcare, and stigma and discrimination at the legal or policy level are eligible. We will exclude publications exclusively addressing community and cultural stigma.

\section{Comparator/study design}

Publications introducing frameworks will be included regardless of the comparator or study design. Measure research needs to describe the tool in sufficient detail to be included but needs no comparator. Experimental evaluations of interventions have to report on a concurrent comparator (eg, randomised controlled trial, controlled clinical trial, comparative studies, quasi-experimental studies, natural experiments) to be eligible. Observational evaluations of healthcare interventions not under the control of the investigator and influenced by secular trends, have to report on a historic or concurrent comparator (eg, pre-post, cohort study comparing two cohorts) and be sufficiently large or encompassing to be eligible (either demonstrating statistical power in a power analysis, reporting on large-scale evaluations of 200 participants or more, or ensuring that all eligible units have been targeted such as all healthcare providers in a hospital received the intervention). For studies evaluating the impacts of laws, no comparator is required if the study author can demonstrate an alternative analysis of determining the effect of the law not compared with a prior period or different legislative environment. Systematic reviews will be retained for an umbrella review providing context and for reference-mining.

\section{Outcome}

Framework publications will be included regardless of any reported outcomes. Measure research needs to report a detailed description of the measure, the development process, or the evaluation or validation of the measure. Intervention research needs to provide a structured evaluation of an indicator of stigma or operationalisation of discrimination to be eligible.

\section{Timing}

Frameworks will not be restricted by publication year. For measures and interventions, only those published from 2008 on will be included, building on the first People Living with HIV Stigma Index published in 2008, which transformed thinking around HIV-related stigma measurement. ${ }^{205}$

\section{Setting}

The review is not restricted regarding setting but we will restrict to English language publications. 


\section{Data abstraction}

For the frameworks, we will abstract the author group and publisher; publication year, scope, aim or purpose of the framework; subtype and definition of the constructs stigma and/or discrimination; addressed targets (eg, people living with HIV and their families); framework components; and a broad summary of the framework. The Draft Evidence Table Key Question 1 will summarise the identified frameworks (see online supplemental appendix 1).

For measures, we will document the author group; publication year; name of the tool; the stigma or discrimination subtype being assessed, the underlying framework (where applicable) and definitions of stigma and discrimination; the targeted population; the surveyed population used to develop or assess the measure; the scale structure of the tool, number of items, and answer mode; the documented reliability; and evidence of validity. Draft Evidence Table Key Question 2 outlines the evidence table documenting this information for each included publication (see online supplemental appendix 1).

For intervention evaluations (key question 3), we will document the study identification details, year of publication, country, study design, sample size, participant details, context or setting; intervention type, intervention description and intervention components, comparator type and comparator description, definition and measures of stigma and discrimination, and findings for the outcome measures. In order to document the effect of the intervention concisely, we will abstract data for the main effectiveness signal and adverse events or unintended consequences. In addition, we will record any information provided by the study authors on the appropriateness of the used measures, intervention or comparator. The draft evidence table is shown in online supplemental appendix 1 (Draft Evidence Table Key Question 3).

\section{Critical appraisal and analysis plan}

For frameworks, we will assess the source (eg, published by an individual author group or endorsement by a professional organisation) and processes used to develop the framework, including stakeholder involvement and formal consensus finding methods. ${ }^{206} 207$

For measures, we will evaluate the demonstrated reliability (internal consistency, test-retest stability, rater agreement) as well as evidence of the validity (eg, content, criterion or construct validity) applying COnsensus-based Standards for the selection of health Measurement Instruments criteria. ${ }^{208}$

Intervention evaluations will be evaluated for potential selection, detection, performance, attrition, reporting and study-specific sources of bias, adapting RoB 2 and ROBINS-I criteria. ${ }^{209} 210$

To determine the effects of the interventions, we will compute measure-independent effect estimates for all included studies, that is, standardised mean differences for continuous outcomes and relative risks for categorical outcomes, to facilitate comparisons across studies. Interpretation of the effect sizes will take the statistical significance of the difference compared with the control or preintervention status into account as well as the statistical power of the study to detect an effect. Where possible, results of studies across interventions will be summarised in random effects meta-analyses applying Hartung-Knapp corrections for small samples. $^{211}$

To explore which interventions are successful and to determine what characterises these interventions, we will first broadly categorise the type of intervention. The categorisation will be undertaken based on the abstracted intervention description and will be blind to the findings of the study. The categories will be used to identify subgroups of more homogeneous intervention types. In a second step, we will derive a set of common intervention components drawn from the identified literature and informed by existing intervention frameworks and taxonomies. The presence or absence of components will be documented for each successful and each unsuccessful intervention, together with context information such as the country of evaluation. The draft component tables for the successful and unsuccessful interventions are shown in online supplemental appendix 1 (Draft Component Table Key Question 4a, Draft Component Table Key Question 4b).

Categorising the evaluations as successful or unsuccessful will follow a transparent algorithm and will be determined by two independent literature reviewers to reduce errors and bias. For all studies, the reduction of stigma will be determined. Assessments will be based on the results in the intervention group relative to a control group. In the absence of a concurrent control group, the change compared with the preintervention status will be used to determine intervention success. Categorising interventions as successful or unsuccessful will qualitatively stratify the identified research. We acknowledge that this step will lose the granularity of a continuous variable. However, we anticipate that a broad categorisation is necessary given the diversity of the approaches. We will use transparent methods to categorise studies and discrepancies in the effect classification between reviewer ratings will be resolved through discussion in the review team.

Determining characteristics of successful interventions and success factors in often complex and multicomponent interventions, as well as reasons for failure, will require a careful analysis of the components or 'active ingredients' of the interventions. We will apply principles of qualitative comparative analysis and review the established component matrix across the successful interventions in seeking to determine which individual and figurations of factors appear to be associated with success. ${ }^{212}$ We will explore differences in the component structure between interventions that were determined to be successful and those that were determined to be unsuccessful to identify components likely be associated with success. We will conduct meta-regressions across studies to confirm effects 
of components. Meta-regressions will add the component of interest to the meta-analytic model to determine whether its presence or absence affects the size of the intervention effect.

\section{Summary of findings and body of evidence assessment}

We will document transparent criteria to evaluate the certainty in the evidence across the included research. We will adapt the eight Grading of Recommendations, Assessment, Development and Evaluation criteria study limitation, inconsistency, imprecision, indirectness, reporting bias to upgrade, and the criteria large effect, dose-response relationship, and confounding would mask an effect, to downgrade the quality of evidence. ${ }^{213}$ While the criterion study limitation is applicable to all key questions, inconsistency (eg, of reliability estimates across studies) will be used to interpret the quality of evidence for key questions 2, 3 and 4, and the criterion imprecision (eg, range of reported reliability estimates) will be applied to key questions 2 and 3. All other criteria will primarily apply to key question 3 and the evidence statements that can be formulated to answer this key question.

The evaluation of the body of evidence will be used to arrive at internationally accepted certainty categories that communicate our confidence in the findings using the categories high, moderate, low and very low. High indicates confidence that the true effect is similar to the estimated effect, moderate indicates the true effect is probably close to the estimated effect, low suggests the true effect might be markedly different from the estimated effect, and very low signals that the true effect is probably markedly different from the estimated effect. We will review the appropriateness of the starting point of low quality of evidence for non-randomised studies before upgrading or downgrading of the evidence to avoid floor effects and ensure meaningful differentiation.

\section{Synthesis}

The identified evidence will be documented in comprehensive tables and figures. The literature flow will be documented in a literature flow diagram and account for all identified research. Critical appraisal of frameworks and measures will be integrated into the evidence tables. The risk of bias across intervention studies will be documented in a risk of bias figure showing the distribution for each criterion. All included research will be documented in concise evidence tables providing details for each included framework, measure and intervention evaluation (see drafts in online supplemental appendix 1).

With this review, we intend to establish a compendium of existing frameworks for HIV-related stigma and discrimination. Frameworks published under Creative Commons licences, and where copyright agreements allow these for other frameworks, will be shown in full to allow a meaningful overview. Where copyright assertions cannot be obtained, the framework evidence table will include the link to the framework if available in the public domain.
The evidence table for measures aims to provide a resource for future HIV stigma and discrimination research. It will provide a comprehensive overview of the available measures to help select tools for future studies.

The evidence table and component tables for the identified interventions aim to document the existing knowledge base for interventions to address self-stigma, stigma and discrimination in healthcare, and stigma and discrimination in laws and policies. A detailed exploration of intervention components aims to support efforts in prevention, reducing and mitigating HIV-related stigma and discrimination.

In addition to a narrative synthesis, we will document the evidence across research in summary of findings tables with one table for each key question. The table summarising key question 1 will document the number and type of the identified stigma and discrimination frameworks. The summary of findings table summarising key question 2 will document the number and type of identified stigma and discrimination measures, listing the measure details and reliability (evidence that the tool is measuring accurately and consistently) and validity (evidence that the tool is measuring what it is supposed to measure). The third table will document the evidence for the interventions (key question 3), organised by context and modality (eg, healthcare facility intervention, law or policy change) and within by outcome category (effectiveness, unintended consequences), together with the quality of evidence assessment. The fourth summary of findings table will document commonalities of successful interventions and failed interventions (key question 4).

\section{Gap analysis}

Part of the systematic review will be a formal gap analysis. We will use a structured approach to document research gaps. Gaps will be documented in a Study design, Participants, Interventions, Framework, Outcomes, Context/ Country framework, with attention to other relevant categories that may emerge during the evidence review. The gap analysis will document research needs and provide concrete recommendations for future research.

\section{Patient and public involvement statement}

The draft protocol was peer-reviewed by international content experts and a representative of the community of persons living with HIV to ensure that the review asks the right questions. Stakeholders will be asked to review the draft review to ensure that all relevant frameworks, measures and intervention evaluations have been captured and that the review contributes meaningfully to the knowledge base and to ensure that the evidence review is as impactful as possible.

\section{Ethics and dissemination}

The systematic review conduct will be transparent and is designed to advance research to support people living with or suspected to be living with HIV and people disproportionally affected by HIV. The review is considered 
exempt as it does not involve human subjects and does not require review by the human subject protection committee.

The review will be registered in PROSPERO, the results will be published in a journal manuscript and presented at research conferences. The data will be made available in the Systematic Review Data Repository. ${ }^{12}$

Acknowledgements We thank Lucy Stackpool-Moore, Tessa Oraro-Lawrence, Kasoka Kasoka, Brent Allan, and members of the country team for helpful comments.

Contributors SH, LF and SG developed the systematic review, all authors (SH, LF, $\mathrm{MB}, \mathrm{SY}, \mathrm{NF}, \mathrm{AM}$ and $\mathrm{SG}$ ) contributed to this protocol. SH serves as guarantor and accepts full responsibility for the work and the conduct of the study, had access to data and controlled the decision to publish. The corresponding author attests that all listed authors meet authorship criteria and that no others meeting the criteria have been omitted.

Funding This work (Global Systematic Evidence Review: Getting to the Heart of Stigma) is supported by the International AIDS Society (IAS). This work was supported, in whole or in part, by the Bill \& Melinda Gates Foundation [Grant Number INV-004364]. Under the grant conditions of the Foundation, a Creative Commons Attribution 4.0 Generic License has already been assigned to the Author Accepted Manuscript version that might arise from this submission.

Disclaimer The funder had no role in the development of the protocol or decision to submit this manuscript.

Competing interests None declared.

Patient consent for publication Not applicable.

Provenance and peer review Not commissioned; externally peer reviewed.

Data availability statement The data will be made available in the Systematic Review Data Repository (https://srdr.ahrq.gov/).

Supplemental material This content has been supplied by the author(s). It has not been vetted by BMJ Publishing Group Limited (BMJ) and may not have been peer-reviewed. Any opinions or recommendations discussed are solely those of the author(s) and are not endorsed by BMJ. BMJ disclaims all liability and responsibility arising from any reliance placed on the content. Where the content includes any translated material, BMJ does not warrant the accuracy and reliability of the translations (including but not limited to local regulations, clinical guidelines, terminology, drug names and drug dosages), and is not responsible for any error and/or omissions arising from translation and adaptation or otherwise.

Open access This is an open access article distributed in accordance with the Creative Commons Attribution 4.0 Unported (CC BY 4.0) license, which permits others to copy, redistribute, remix, transform and build upon this work for any purpose, provided the original work is properly cited, a link to the licence is given, and indication of whether changes were made. See: https://creativecommons.org/ licenses/by/4.0/.

ORCID iD

Susanne Hempel http://orcid.org/0000-0003-1597-5110

\section{REFERENCES}

1 Goffman E. Stigma: notes on the management of spoiled identity. Englewood Cliffs, New Jersey: Prentice-Hall, 1963.

2 Hatzenbuehler ML, Phelan JC, Link BG. Stigma as a fundamental cause of population health inequalities. Am J Public Health 2013;103:813-21.

3 Relf MV, L Holzemer W, Holt L, et al. A review of the state of the science of HIV and stigma: context, conceptualization, measurement, interventions, gaps, and future priorities. J Assoc Nurses AIDS Care 2021;32:392-407.

4 Parker R, Aggleton P, . Hiv and AIDS-related stigma and discrimination: a conceptual framework and implications for action. Soc Sci Med 2003;57:13-24.

5 Joint United Nations Programme on HIV/AIDS. Reduction of HIV-related stigma and discrimination: guidance note. Geneva, Switzerland: UNAIDS, 2014.
6 UN General Assembly. Declaration of human rights 10 December 1948, 217 A (III), 1948. Available: https://www.refworld.org/docid/ 3ae6b3712c.html [Accessed 29 Aug 2021].

7 Pantelic M, Steinert Jl, Park J, et al. 'Management of a spoiled identity': systematic review of interventions to address self-stigma among people living with and affected by HIV. BMJ Glob Health 2019:4:e001285.

8 UNAIDS. Evidence for eliminating HIV-related stigma and discrimination: joint United nations programme on HIV/AIDS (UNAIDS), 2020. Available: https://www.unaids.org/en/resources/ documents/2020/eliminating-discrimination-guidance

9 UNAIDS. Global AIDS strategy 2021-2026 - end inequalities. In: End AIDS. Geneva, Switzerland: UNAIDS, 2021.

10 United Nations General Assembly. Political Declaration on HIV and AIDS: ending inequalities and getting on track to end AIDS by 2030. A/RES/75/284. New York, USA: United Nations, 2021.

11 UNAIDS. Mapping of tools on HIV-related stigma and discrimination in health care settings: final report, 2015. Available: http://teampata.org/wp-content/uploads/2017/06/Mapping-oftools-on-HIV-related-stigma-and-discrimination-in-health-caresettings.pdf

12 Agency for Healthcare Research and Quality. SRDR systematic review data reposity. Available: https://srdr.ahrq.gov/ [Accessed 11 May 2021].

13 Amare T, Getinet W, Shumet S, et al. Prevalence and associated factors of depression among PLHIV in Ethiopia: systematic review and meta-analysis, 2017. AIDS Res Treat 2018;2018:5462959.

14 Ayano G, Solomon M, Abraha M. A systematic review and metaanalysis of epidemiology of depression in people living with HIV in East Africa. BMC Psychiatry 2018;18:254.

15 Bayat A-H, Mohammadi R, Moradi-Joo M, et al. Hiv and drug related stigma and risk-taking behaviors among people who inject drugs: a systematic review and meta-analysis. J Addict Dis 2020;38:71-83.

16 Belayneh Z, Mekuriaw B, Mehare T, et al. Magnitude and predictors of common mental disorder among people with HIV/AIDS in Ethiopia: a systematic review and meta-analysis. BMC Public Health 2020;20:689.

17 Dessauvagie AS, Jörns-Presentati A, Napp A-K, et al. The prevalence of mental health problems in sub-Saharan adolescents living with HIV: a systematic review. Glob Ment Health 2020;7:e29.

18 Evangeli M, Ferris K, Kenney NM, et al. A systematic review of psychological correlates of HIV testing intention. AIDS Care 2018;30:18-26.

19 Gesesew HA, Tesfay Gebremedhin A, Demissie TD, et al. Significant association between perceived HIV related stigma and late presentation for HIV/AIDS care in low and middle-income countries: a systematic review and meta-analysis. PLoS One 2017;12:e0173928.

20 Ghiasvand H, Higgs P, Noroozi M, et al. Social and demographical determinants of quality of life in people who live with HIV/AIDS infection: evidence from a meta-analysis. Biodemography Soc Biol 2020;65:57-72.

21 Govindasamy D, Seeley J, Olaru ID, et al. Informing the measurement of wellbeing among young people living with HIV in sub-Saharan Africa for policy evaluations: a mixed-methods systematic review. Health Qual Life Outcomes 2020;18:120.

22 Griswold MK, Pagano-Therrien J. Women living with HIV in high income countries and the deeper meaning of breastfeeding avoidance: a metasynthesis. J Hum Lact 2020;36:44-52.

23 Haines C, Loades ME, Coetzee BJ, et al. Which HIV-infected youth are at risk of developing depression and what treatments help? A systematic review focusing on southern Africa. Int J Adolesc Med Health 2019. doi:10.1515/ijamh-2019-0037. [Epub ahead of print: 06 Aug 2019].

24 Ishola F. P4.41 effect of HIV related stigma on utilisation of skilled birth attendants by HIV positive women in Nigeria; a systematic review. Sex Transm Infect 2017;93. doi:10.1136/ sextrans-2017-053264.538

$25 \mathrm{Li} \mathrm{H}$, Li X, Zhang L, et al. Effects of multiple types of stigma on the probability of HIV disclosure to sex partners: a systematic review. Sex Health 2016;13:516-29.

26 MacCarthy S, Poteat T, Xia Z, et al. Current research gaps: a global systematic review of HIV and sexually transmissible infections among transgender populations. Sex Health 2017;14:456-68.

27 MacLean JR, Wetherall K. The association between HIV-Stigma and depressive symptoms among people living with HIV/AIDS: a systematic review of studies conducted in South Africa. J Affect Disord 2021;287:125-37.

28 Magno L, Silva LAVda, Veras MA, et al. Stigma and discrimination related to gender identity and vulnerability to HIV/AIDS among 
transgender women: a systematic review. Cad Saude Publica 2019;35:e00112718.

29 Narasimhan M, Payne C, Caldas S, et al. Ageing and healthy sexuality among women living with HIV. Reprod Health Matters 2016;24:43-51.

30 Necho M, Belete A, Tsehay M. Depressive symptoms and their determinants in patients who are on antiretroviral therapy in the case of a low-income country, Ethiopia: a systematic review and meta-analysis. Int J Ment Health Syst 2021;15:3.

31 Necho M, Tsehay M, Zenebe Y. Suicidal ideation, attempt, and its associated factors among HIV/AIDS patients in Africa: a systematic review and meta-analysis study. Int J Ment Health Syst 2021;15:13.

32 Pantelic M, Shenderovich Y, Cluver L, et al. Predictors of internalised HIV-related stigma: a systematic review of studies in sub-Saharan Africa. Health Psychol Rev 2015;9:469-90.

33 Rueda S, Mitra S, Chen S, et al. Examining the associations between HIV-related stigma and health outcomes in people living with HIV/AIDS: a series of meta-analyses. BMJ Open 2016;6:e011453.

34 Rzeszutek M, Gruszczyńska E. Posttraumatic growth among people living with HIV: a systematic review. J Psychosom Res 2018;114:81-91.

35 Scofield D, Moseholm E. Hiv-elated stigma and health-related quality of life in women living with HIV in developed countries: a systematic review. AIDS Care 2021;9:1-9.

36 Scott W, Arkuter C, Kioskli K, et al. Psychosocial factors associated with persistent pain in people with HIV: a systematic review with meta-analysis. Pain 2018;159:2461-76.

37 Yassin Z, Erasmus C, Frantz J. HIV-related stigma and the psychosocial well-being of children orphaned by AIDS: a systematic review. Vulnerable Child Youth Stud 2018;13:247-58.

38 Yassin Z, Erasmus CJ. The impact of HIV-related stigma on the psychological well-being of children who have been orphaned by AIDS. Vulnerable Child Youth Stud 2016;11:297-323.

39 Zgambo M, Kalembo FW, Mbakaya BC. Risky behaviours and their correlates among adolescents living with HIV in sub-Saharan Africa: a systematic review. Reprod Health 2018;15:180.

40 Zhao Y, Luo T, Tucker JD, et al. Risk factors of HIV and other sexually transmitted infections in China: a systematic review of reviews. PLoS One 2015;10:e0140426.

41 Aggarwal NK, Consavage KE, Dhanuka I, et al. Health and health care access barriers among transgender women engaged in sex work: a synthesis of U.S.-Based studies published 2005-2019. LGBT Health 2021;8:11-25.

42 Ahmed S, Autrey J, Katz IT, et al. Why do people living with HIV not initiate treatment? a systematic review of qualitative evidence from low- and middle-income countries. Soc Sci Med 2018;213:72-84.

43 Bass SB, D'Avanzo P, Alhajji M, et al. Exploring the engagement of racial and ethnic minorities in HIV treatment and vaccine clinical trials: a scoping review of literature and implications for future research. AIDS Patient Care STDS 2020;34:399-416.

44 Brookfield S, Dean J, Forrest C, et al. Barriers to accessing sexual health services for transgender and male sex workers: a systematic qualitative Meta-summary. AIDS Behav 2020;24:682-96.

45 Ferguson L, Grant AD, Watson-Jones D, et al. Linking women who test HIV-positive in pregnancy-related services to long-term HIV care and treatment services: a systematic review. Trop Med Int Health 2012;17:564-80.

46 Ganle JK, Baatiema L, Quansah R, et al. Barriers facing persons with disability in accessing sexual and reproductive health services in sub-Saharan Africa: a systematic review. PLoS One 2020;15:19.

47 Gari S, Doig-Acuña C, Smail T, et al. Access to HIV/AIDS care: a systematic review of socio-cultural determinants in low and high income countries. BMC Health Serv Res 2013;13:198.

48 Gonçalves TR, Costa AHC, Sales MS, et al. [Combined HIV prevention? systematic review of interventions with women from low- and middle-income countries]. Cien Saude Colet 2020;25:1897-912.

49 Gourlay A, Birdthistle I, Mburu G, et al. Barriers and facilitating factors to the uptake of antiretroviral drugs for prevention of mother-to-child transmission of HIV in sub-Saharan Africa: a systematic review. J Int AIDS Soc 2013;16:18588.

50 Govindasamy D, Ford N, Kranzer K. Risk factors, barriers and facilitators for linkage to antiretroviral therapy care: a systematic review. AIDS 2012;26:2059-67.

51 Hodgson I, Plummer ML, Konopka SN, et al. A systematic review of individual and contextual factors affecting art initiation, adherence, and retention for HIV-infected pregnant and postpartum women. PLoS One 2014;9:e111421.

52 Jones C, Ritchwood TD, Taggart T. Barriers and facilitators to the successful transition of adolescents living with HIV from pediatric to adult care in low and middle-income countries: a systematic review and policy analysis. AIDS Behav 2019;23:2498-513.

53 Medved Kendrick H, Kendrick HM. Are religion and spirituality barriers or facilitators to treatment for HIV: a systematic review of the literature. AIDS Care 2017;29:1-13.

54 Lancaster KE, Cernigliaro D, Zulliger R, et al. HIV care and treatment experiences among female sex workers living with HIV in sub-Saharan Africa: a systematic review. Afr J AIDS Res 2016;15:377-86.

55 Matacotta JJ, Rosales-Perez FJ, Carrillo CM. HIV preexposure prophylaxis and treatment as prevention - beliefs and access barriers in men who have sex with men (msm) and transgender women: a systematic review. J Patient Cent Res Rev 2020;7:265-74.

56 Mathias A, Alves Dos Santos L, Grangeiro A, et al. Thematic synthesis HIV prevention qualitative studies in men who have sex with men (MSM). Colomb Med 2019;50:201-14.

57 Mwaturura C, Traeger M, Lemoh C, et al. Barriers and facilitators to pre-exposure prophylaxis among African migrants in high income countries: a systematic review. Sex Health 2021;18:130-9.

58 O'Brien N, Hong QN, Law S, et al. Health system features that enhance access to comprehensive primary care for women living with HIV in high-income settings: a systematic mixed studies review. AIDS Patient Care STDS 2018;32:129-48.

59 Pellowski JA. Barriers to care for rural people living with HIV: a review of domestic research and health care models. J Assoc Nurses AIDS Care 2013;24:422-37.

60 Peng P, Su S, Fairley CK, et al. A global estimate of the acceptability of pre-exposure prophylaxis for HIV among men who have sex with men: a systematic review and meta-analysis. AIDS Behav 2018;22:1063-74.

61 Rade DA, Crawford G, Lobo R, et al. Sexual health help-seeking behavior among migrants from sub-Saharan Africa and South East Asia living in high income countries: a systematic review. Int J Environ Res Public Health 2018;15. doi:10.3390/ijerph15071311. [Epub ahead of print: 2206 2018].

62 Russ S, Zhang C, Liu Y. Pre-exposure prophylaxis care continuum, barriers, and facilitators among black men who have sex with men in the United States: a systematic review and meta-analysis. AIDS Behav 2021;25:2278-88

63 Scholl E. Improving outpatient implementation of preexposure prophylaxis in men who have sex with men. J Am Assoc Nurse Pract 2016;28:446-52.

64 Wang Z, Yuan T, Fan S, et al. HIV nonoccupational postexposure prophylaxis among men who have sex with men: a systematic review and meta-analysis of global data. AIDS Patient Care STDS 2020;34:193-204.

65 Wao H, Aluoch M, Odondi GO, et al. MSM's versus health care providers' perceptions of barriers to uptake of HIV/AIDS-related interventions: systematic review and meta-synthesis of qualitative and quantitative evidence. Int J Sex Health 2016;28:151-62.

66 Young T, Busgeeth K. Home-based care for reducing morbidity and mortality in people infected with HIV/AIDS. Cochrane Database Syst Rev 2010;1:Cd005417.

67 Alvarez-del Arco D, Monge S, Azcoaga A, et al. HIV testing and counselling for migrant populations living in high-income countries: a systematic review. Eur J Public Health 2013;23:1039-45.

68 Blackstone SR, Nwaozuru U, Iwelunmor J. Antenatal HIV testing in sub-Saharan Africa during the implementation of the millennium development goals: a systematic review using the PEN-3 cultural model. Int Q Community Health Educ 2018;38:115-28.

69 Bolsewicz K, Vallely A, Debattista J, et al. Factors impacting HIV testing: a review--perspectives from Australia, Canada, and the UK. AIDS Care 2015;27:570-80.

70 Campbell CK, Lippman SA, Moss N, et al. Strategies to increase HIV testing among MSM: a synthesis of the literature. AIDS Behav 2018;22:2387-412

71 Chamla D, Luo C, Adjorlolo-Johnson G, et al. Integration of HIV infant testing into immunization programmes: a systematic review. Paediatr Int Child Health 2015;35:298-304.

72 Duffy M, Sharer M, Berhan A, et al. Hiv risk screening for high-yield community testing services for orphans and vulnerable children: a literature review. Vulnerable Child Youth Stud 2018;13:95-115.

73 Evangeli M, Pady K, Wroe AL. Which psychological factors are related to HIV testing? a quantitative systematic review of global studies. AIDS Behav 2016;20:880-918.

74 Feyissa GT, Lockwood C, Munn Z. The effectiveness of homebased HIV counseling and testing on reducing stigma and risky sexual behavior among adults and adolescents: a systematic review and meta-analyses. JBI Database System Rev Implement Rep 2015;13:318-72. 
75 Hamilton A, Shin S, Taggart T, et al. HIV testing barriers and intervention strategies among men, transgender women, female sex workers and incarcerated persons in the Caribbean: a systematic review. Sex Transm Infect 2020;96:189-96.

76 Hamilton A, Thompson N, Choko AT, et al. HIV self-testing uptake and intervention strategies among men in sub-Saharan Africa: a systematic review. Front Public Health 2021;9:594298.

77 Krause J, Subklew-Sehume F, Kenyon C, et al. Acceptability of HIV self-testing: a systematic literature review. BMC Public Health 2013;13:735

78 Laprise C, Bolster-Foucault C. Understanding barriers and facilitators to HIV testing in Canada from 2009-2019: a systematic mixed studies review. Can Commun Dis Rep 2021;47:105-25.

79 Leidel S, Wilson S, McConigley R, et al. Health-care providers' experiences with opt-out HIV testing: a systematic review. AIDS Care 2015;27:1455-67.

80 Lorenc T, Marrero-Guillamón I, Llewellyn A, et al. HIV testing among men who have sex with men (MSM): systematic review of qualitative evidence. Health Educ Res 2011;26:834-46.

81 Musheke M, Ntalasha H, Gari S, et al. A systematic review of qualitative findings on factors enabling and deterring uptake of HIV testing in sub-Saharan Africa. BMC Public Health 2013;13:220.

82 Nnko S, Kuringe E, Nyato D, et al. Determinants of access to HIV testing and counselling services among female sex workers in sub-Saharan Africa: a systematic review. BMC Public Health 2019;19:15.

83 Pant Pai N, Sharma J, Shivkumar S, et al. Supervised and unsupervised self-testing for HIV in high- and low-risk populations: a systematic review. PLoS Med 2013;10:e1001414.

84 Pottie K, Medu O, Welch V, et al. Effect of rapid HIV testing on HIV incidence and services in populations at high risk for HIV exposure: an equity-focused systematic review. BMJ Open 2014;4:e006859.

85 Qiao S, Zhou G, Li X, . Disclosure of same-sex behaviors to healthcare providers and uptake of HIV testing for men who have sex with men: a systematic review. Am J Mens Health 2018;12:1197-214.

86 Qin Y, Han L, Babbitt A, et al. Experiences using and organizing HIV self-testing. AIDS 2018;32:371-81.

87 Stannah J, Dale E, Elmes J, et al. Hiv testing and engagement with the HIV treatment cascade among men who have sex with men in Africa: a systematic review and meta-analysis. Lancet HIV 2019;6:e769-87.

88 Stojanovski K, Naja-Riese G, King EJ, et al. A systematic review of the social network strategy to optimize HIV testing in key populations to end the epidemic in the United States. AIDS Behav 2021;25:1-19.

89 Tan K, Black BP. A systematic review of health care ProviderPerceived barriers and facilitators to routine HIV testing in primary care settings in the southeastern United States. J Assoc Nurses AIDS Care 2018;29:357-70.

90 Tokar A, Broerse JEW, Blanchard J, et al. HIV testing and counseling among female sex workers: a systematic literature review. AIDS Behav 2018;22:2435-57.

91 Witzel TC, Lora W, Lees S, et al. Uptake contexts and perceived impacts of HIV testing and counselling among adults in East and southern Africa: a meta-ethnographic review. PLoS One 2017;12:e0170588.

92 Adeoye-Agboola DI, Evans $\mathrm{H}$, Hewson D, et al. Factors influencing HIV disclosure among people living with HIV/AIDS in Nigeria: a systematic review using narrative synthesis and meta-analysis. Public Health 2016;136:13-28.

93 Azétsop J, Diop BA. Access to antiretroviral treatment, issues of well-being and public health governance in Chad: what justifies the limited success of the universal access policy? Philos Ethics Humanit Med 2013;8:14.

94 Belay GM, Endalamaw A, Ayele AD. Late presentation of HIV positive adults and its predictors to HIV/AIDS care in Ethiopia: a systematic review and meta-analysis. BMC Infect Dis 2019;19:534.

95 Bharat S. A systematic review of HIV/AIDS-related stigma and discrimination in India: current understanding and future needs. Sahara J 2011:8:138-49.

96 Bradley E, Forsberg K, Betts JE, et al. Factors affecting preexposure prophylaxis implementation for women in the United States: a systematic review. J Womens Health 2019;28:1272-85.

97 Darlington CK, Hutson SP. Understanding HIV-related stigma among women in the southern United States: a literature review. AIDS Behav 2017;21:12-26.

98 Das S, Leibowitz GS. Mental health needs of people living with HIV/ AIDS in India: a literature review. AIDS Care 2011;23:417-25.

99 Kerr ZY, Miller KR, Galos D, et al. Challenges, coping strategies, and recommendations related to the HIV services field in the HAART era: a systematic literature review of qualitative studies from the United States and Canada. AIDS Patient Care STDS 2013;27:85-95.

100 Loutfy M, Tharao W, Logie C, et al. Systematic review of stigma reducing interventions for African/black diasporic women. J Int AIDS Soc 2015;18:19835.

101 Mey A, Plummer D, Dukie S, et al. Motivations and barriers to treatment uptake and adherence among people living with HIV in Australia: a mixed-methods systematic review. AIDS Behav 2017;21:352-85.

102 Monteiro SS, Brigeiro M, Vilella WV, et al. Challenges facing HIV treatment as prevention in Brazil: an analysis drawing on literature on testing. Cien Saude Colet 2019;24:1792-806.

103 Sangaramoorthy T, Jamison A, Dyer T. Older African Americans and the HIV care continuum: a systematic review of the literature, 20032018. AIDS Behav 2019;23:973-83.

104 Vaitses Fontanari AM, Zanella GI, Feijó M, et al. Hiv-related care for transgender people: a systematic review of studies from around the world. Soc Sci Med 2019;230:280-94.

105 Weihs M, Meyer-Weitz A. Barriers to workplace HIV testing in South Africa: a systematic review of the literature. AIDS Care 2016;28:495-9.

106 Weldesenbet AB, Kebede SA, Tusa BS. The effect of poor social support on depression among HIV/AIDS patients in Ethiopia: a systematic review and meta-analysis. Depress Res Treat 2020;2020:6633686.

107 Whembolua G-L, Conserve DF, Thomas K, et al. Hiv serostatus disclosure in the Democratic Republic of the Congo: a systematic review. AIDS Care 2019;31:489-93.

108 Wirtz AL, Kirey A, Peryskina A, et al. Uncovering the epidemic of HIV among men who have sex with men in central Asia. Drug Alcohol Depend 2013;132 Suppl 1:S17-24.

109 Young C. Understanding HIV-related posttraumatic stress disorder in South Africa: a review and conceptual framework. Afr J AIDS Res 2011;10:139-48.

110 Ammon N, Mason S, Corkery JM. Factors impacting antiretroviral therapy adherence among human immunodeficiency virus-positive adolescents in sub-Saharan Africa: a systematic review. Public Health 2018;157:20-31.

111 Campbell L, Masquillier C, Thunnissen E, et al. Social and structura determinants of household support for art adherence in low- and middle-income countries: a systematic review. Int $J$ Environ Res Public Health 2020;17:3808.

112 Edeza A, Karina Santamaria E, Valente PK, et al. Experienced barriers to adherence to pre-exposure prophylaxis for HIV prevention among MSM: a systematic review and metaethnography of qualitative studies. AIDS Care 2021;33:1-9.

113 Geter A, Sutton MY, Hubbard McCree D. Social and structural determinants of HIV treatment and care among black women living with HIV infection: a systematic review: 2005-2016. AIDS Care 2018;30:409-16.

114 Heestermans T, Browne JL, Aitken SC, et al. Determinants of adherence to antiretroviral therapy among HIV-positive adults in sub-Saharan Africa: a systematic review. BMJ Glob Health 2016;1:e000125.

115 Katz IT, Ryu AE, Onuegbu AG, et al. Impact of HIV-related stigma on treatment adherence: systematic review and meta-synthesis. J Int AIDS Soc 2013:16:18640.

116 Knettel BA, Cichowitz C, Ngocho JS, et al. Retention in HIV care during pregnancy and the postpartum period in the option $\mathrm{B}+$ era: systematic review and meta-analysis of studies in Africa. J Acquir Immune Defic Syndr 2018;77:427-38.

117 Lambert CC, Mugavero MJ, Najjar YS, et al. The state of adherence to HIV care in black women. J Assoc Nurses AIDS Care 2018;29:487-503

118 Makanjuola T, Taddese HB, Booth A. Factors associated with adherence to treatment with isoniazid for the prevention of tuberculosis amongst people living with HIV/AIDS: a systematic review of qualitative data. PLoS One 2014;9:e87166.

119 Maxwell S, Gafos M, Shahmanesh M. Pre-exposure prophylaxis use and medication adherence among men who have sex with men: a systematic review of the literature. J Assoc Nurses AIDS Care 2019;30:e38-61.

120 Omonaiye O, Kusljic S, Nicholson P, et al. Medication adherence in pregnant women with human immunodeficiency virus receiving antiretroviral therapy in sub-Saharan Africa: a systematic review. BMC Public Health 2018;18:805.

121 Paramesha AE, Chacko LK. Predictors of adherence to antiretroviral therapy among PLHIV. Indian J Public Health 2019;63:367.

122 Shubber Z, Ford N. Adherence to HIV post-exposure prophylaxis for children/adolescents who have been sexually assaulted: a 
systematic review of barriers, enablers, and interventions. Child Abuse Negl 2021;116:104143.

123 Shubber Z, Mills EJ, Nachega JB, et al. Patient-reported barriers to adherence to antiretroviral therapy: a systematic review and metaanalysis. PLoS Med 2016;13:e1002183.

124 Sidebottom D, Ekström AM, Strömdahl S. A systematic review of adherence to oral pre-exposure prophylaxis for HIV - how can we improve uptake and adherence? BMC Infect Dis 2018;18:581.

125 Sweeney SM, Vanable PA. The association of HIV-related stigma to HIV medication adherence: a systematic review and synthesis of the literature. AIDS Behav 2016;20:29-50.

126 Wasti SP, van Teijlingen E, Simkhada P, et al. Factors influencing adherence to antiretroviral treatment in Asian developing countries: a systematic review. Trop Med Int Health 2012;17:71-81.

127 Zuniga JA, Wright C, Fordyce J, et al. Self-management of HIV and diabetes in African American women: a systematic review of qualitative literature. Diabetes Educ 2018;44:419-34.

128 Arias-Colmenero T, Pérez-Morente $\mathrm{M}^{\mathrm{a}}$ Ángeles, Ramos-Morcillo AJ, et al. Experiences and attitudes of people with HIV/AIDS: a systematic review of qualitative studies. Int J Environ Res Public Health 2020;17. doi:10.3390/ijerph17020639. [Epub ahead of print: 1901 2020].

129 Brömdal A, Mullens AB, Phillips TM, et al. Experiences of transgender prisoners and their knowledge, attitudes, and practices regarding sexual behaviors and HIV/STIs: a systematic review. Int $J$ Transgend 2019;20:4-20

130 Chi P, Li X. Impact of parental HIV/AIDS on children's psychological well-being: a systematic review of global literature. AIDS Behav 2013;17:2554-74.

131 Eshun-Wilson I, Rohwer A, Hendricks L, et al. Being HIV positive and staying on antiretroviral therapy in Africa: a qualitative systematic review and theoretical model. PLoS One 2019;14:e0210408.

132 Gannon BN, Stacciarini J-MR. Review of the literature: a rural-urban comparison of social networks of older adults living with HIV. $J$ Assoc Nurses AIDS Care 2016;27:419-29.

133 Goldberg RE, Short SE. What do we know about children living with HIV-infected or AIDS-ill adults in sub-Saharan Africa? a systematic review of the literature. AIDS Care 2016;28 Suppl 2:130-41.

134 Hainsworth EG, Shahmanesh M, Stevenson F. Exploring the views and experiences of HIV positive patients treated for cancer: a systematic review of the literature. AIDS Care 2018;30:535-43.

135 Kimera E, Vindevogel S, De Maeyer J, et al. Challenges and support for quality of life of youths living with HIV/AIDS in schools and larger community in East Africa: a systematic review. Syst Rev 2019;8:64.

136 Lowther K, Selman L, Harding R, et al. Experience of persistent psychological symptoms and perceived stigma among people with HIV on antiretroviral therapy (art): a systematic review. Int J Nurs Stud 2014:51:1171-89.

137 Mclnerney P, Brysiewicz P. A systematic review of the experiences of caregivers in providing home-based care to persons with HIV/ AIDS in Africa. JBI Libr Syst Rev 2009;7:130-53.

138 Nattabi B, Li J, Thompson SC, et al. A systematic review of factors influencing fertility desires and intentions among people living with HIV/AIDS: implications for policy and service delivery. AIDS Behav 2009;13:949-68.

139 Roger KS, Mignone J, Kirkland S. Social aspects of HIV/AIDS and aging: a thematic review. Can J Aging 2013;32:298-306.

140 Rubtsova AA, Kempf M-C, Taylor TN, et al. Healthy aging in older women living with HIV infection: a systematic review of psychosocial factors. Curr HIVIAIDS Rep 2017;14:17-30.

141 Zeglin RJ, Hergenrather KC, Garcia JH. A systematic review of the common experiences of caregivers of HIV plus men who have sex with men: implications for Counselors. $J$ Rehabil 2018;84:22-30 https://www.thefreelibrary.com/A+Systematic+ Review+of+the+Common+Experiences+of+Caregivers+of+HIV\% 2B.-a0537853430

142 Baral S, Holland CE, Shannon K, et al. Enhancing benefits or increasing harms: community responses for HIV among men who have sex with men, transgender women, female sex workers, and people who inject drugs. J Acquir Immune Defic Syndr 2014;66 Suppl 3:S319-28.

143 Beres LK, Narasimhan M, Robinson J, et al. Non-specialist psychosocial support interventions for women living with HIV: a systematic review. AIDS Care 2017;29:1079-87.

144 Campbell C, Skovdal M, Gibbs A. Creating social spaces to tackle AIDS-related stigma: reviewing the role of church groups in subSaharan Africa. AIDS Behav 2011:15:1204-19.

$145 \mathrm{Han} \mathrm{H}-\mathrm{R}, \mathrm{Kim} \mathrm{K}$, Murphy J, et al. Community health worker interventions to promote psychosocial outcomes among people living with HIV-A systematic review. PLoS One 2018;13:e0194928.
146 Kaufman ZA, Spencer TS, Ross DA. Effectiveness of sport-based HIV prevention interventions: a systematic review of the evidence. AIDS Behav 2013;17:987-1001.

147 Kerrigan D, Kennedy CE, Morgan-Thomas R, et al. A community empowerment approach to the HIV response among sex workers: effectiveness, challenges, and considerations for implementation and scale-up. Lancet 2015;385:172-85.

148 Mak WWS, Mo PKH, Ma GYK, et al. Meta-analysis and systematic review of studies on the effectiveness of HIV stigma reduction programs. Soc Sci Med 2017;188:30-40.

149 Rao D, Elshafei A, Nguyen M, et al. A systematic review of multilevel stigma interventions: state of the science and future directions. BMC Med 2019;17:41.

150 Sengupta S, Banks B, Jonas D, et al. HIV interventions to reduce HIV/AIDS stigma: a systematic review. AIDS Behav 2011;15:1075-87

151 Skevington SM, Sovetkina EC, Gillison FB. A systematic review to quantitatively evaluate 'stepping stones': a participatory community-based HIV/AIDS prevention intervention. AIDS Behav 2013;17:1025-39.

152 Stangl AL, Lloyd JK, Brady LM, et al. A systematic review of interventions to reduce HIV-related stigma and discrimination from 2002 to 2013: how far have we come? J Int AIDS Soc 2013;16:18734.

153 Williams MV, Palar K, Derose KP. Congregation-based programs to address HIV/AIDS: elements of successful implementation. J Urban Health 2011;88:517-32.

154 Aderomilehin O, Hanciles-Amu A, Ozoya OO. Perspectives and practice of HIV disclosure to children and adolescents by healthcare providers and caregivers in sub-Saharan Africa: a systematic review. Front Public Health 2016:4:166.

155 Cao W, Wong HM, Chang C, et al. Behavioral interventions promoting HIV serostatus disclosure to sex partners among HIVpositive men who have sex with men: a systematic review. Int $J$ Public Health 2019;64:985-98.

156 Kennedy CE, Haberlen S, Amin A, et al. Safer disclosure of HIV serostatus for women living with HIV who experience or fear violence: a systematic review. J Int AIDS Soc 2015;18:20292. 20292.

157 Krauss BJ, Letteney S, Okoro CN. Why tell children: a synthesis of the global literature on reasons for disclosing or not disclosing an HIV diagnosis to children 12 and under. Front Public Health 2016;4:13.

158 Qiao S, Li X, Stanton B. Disclosure of parental HIV infection to children: a systematic review of global literature. AIDS Behav 2013;17:369-89.

159 Tam M, Amzel A, Phelps BR. Disclosure of HIV serostatus among pregnant and postpartum women in sub-Saharan Africa: a systematic review. AIDS Care 2015;27:436-50.

160 Vreeman RC, Gramelspacher AM, Gisore PO, et al. Disclosure of HIV status to children in resource-limited settings: a systematic review. J Int AIDS Soc 2013;16:18466. 18466.

161 Whembolua G-L, Conserve DF, Thomas K, et al. A systematic review of HIV serostatus disclosure among African immigrants in Europe. J Immigr Minor Health 2017;19:947-58.

162 Wilkinson B, Arora KS. A systematic review on confidentiality, disclosure, and stigma in the United States: lessons for HIV care in pregnancy from reproductive genetics. New Bioeth 2015;21:142-54.

163 Cheema E, Abbas A, Al-Hamid A. Healthcare-related factors affecting the management of HIV infected patients: a systematic review of qualitative evidence. Int J STD AIDS 2019;30:1350-61.

164 Feyissa GT, Lockwood C, Woldie M, et al. Reducing HIV-related stigma and discrimination in healthcare settings: a systematic review of guidelines, tools, standards of practice, best practices, consensus statements and systematic reviews. $J$ Multidiscip Healthc 2018;11:405-16.

165 Feyissa GT, Lockwood C, Woldie M, et al. Reducing HIV-related stigma and discrimination in healthcare settings: a systematic review of quantitative evidence. PLoS One 2019:14:e0211298.

166 Geter A, Herron AR, Sutton MY. HIV-Related stigma by healthcare providers in the United States: a systematic review. AIDS Patient Care STDS 2018;32:418-24.

167 Kaladharan S, Daken K, Mullens AB, et al. Tools to measure HIV knowledge, attitudes \& practices (KAPs) in healthcare providers: a systematic review. AIDS Care 2020:1-7.

168 Nyblade L, Stockton MA, Giger K, et al. Stigma in health facilities: why it matters and how we can change it. BMC Med 2019;17:25.

169 Phillips JC, Caine V, Dewart G, et al. Teaching HIV-specific content for pre-licensure nursing and health professions students: a review and synthesis. AIDS Care 2018;30:1614-21. 
170 Pleuhs B, Quinn KG, Walsh JL, et al. Health care provider barriers to HIV pre-exposure prophylaxis in the United States: a systematic review. AIDS Patient Care STDS 2020;34:111-23.

171 Anderson JE, Kanters S. Lack of sexual minorities' rights as a barrier to HIV prevention among men who have sex with men and transgender women in Asia: a systematic review. LGBT Health 2015;2:16-26.

172 Juliastuti D, Dean J, Fitzgerald L. Sexual and reproductive health of women living with HIV in Muslim-majority countries: a systematic mixed studies review. BMC Int Health Hum Rights 2020;20:5.

173 Jürgens R, Csete J, Amon JJ, et al. People who use drugs, HIV, and human rights. The Lancet 2010;376:475-85.

174 Malta M, Ralil da Costa M, Bastos FI. The paradigm of universal access to HIV-treatment and human rights violation: how do we treat HIV-positive people who use drugs? Curr HIVIAIDS Rep 2014;11:52-62.

175 Oldenburg CE, Perez-Brumer AG, Reisner SL, et al. Human rights protections and HIV prevalence among MSM who sell sex: crosscountry comparisons from a systematic review and meta-analysis. Glob Public Health 2018;13:414-25.

176 Stangl AL, Singh D, Windle M, et al. A systematic review of selected human rights programs to improve HIV-related outcomes from 2003 to 2015: what do we know? BMC Infect Dis 2019;19:209.

177 , Wagener MN, Roelofs P, et al, Dutch Institute for health care impruvement. The development of a multidisciplinary, evidence-based guideline for "HIV and employment". AIDS Care 2015;27:133-41.

178 Chambers LA, Wilson MG, Rueda S, et al. Evidence Informing the intersection of HIV, aging and health: a scoping review. AIDS Behav 2014;18:661-75.

179 Ingram L, Stafford C, Deming ME, et al. A systematic mixed studies review of the Intersections of social-ecological factors and HIV stigma in people living with HIV in the U.S. South. J Assoc Nurses AIDS Care 2019;30:330-43.

180 Jackson-Best F, Edwards N. Stigma and intersectionality: a systematic review of systematic reviews across HIV/AIDS, mental illness, and physical disability. BMC Public Health 2018;18:919.

181 Monteiro SS, Villela WV, Soares PS. The interaction between axes of inequality in studies on discrimination, stigma and HIV/AIDS: contributions to the recent international literature. Glob Public Health 2013;8:519-33.

182 Smith SC. The intersection of race, military status, and disability: a systematic review of marginalization and self-esteem being a mediator against negative life outcomes for HIV positive African American male veterans [PsyD.] Azusa Pacific University; 2019.

183 Wilson PA, Valera P, Martos AJ, et al. Contributions of qualitative research in informing HIV/AIDS interventions targeting black MSM in the United States. J Sex Res 2016;53:642-54.

184 Alexandra Marshall S, Brewington KM, Kathryn Allison M, et al. Measuring HIV-related stigma among healthcare providers: a systematic review. AIDS Care 2017;29:1337-45.

185 Earnshaw VA, Chaudoir SR. From conceptualizing to measuring HIV stigma: a review of HIV stigma mechanism measures. AIDS Behav 2009;13:1160-77

186 Fitzgerald-Husek A, Van Wert MJ, Ewing WF, et al. Measuring stigma affecting sex workers (SW) and men who have sex with men (MSM): a systematic review. PLoS One 2017;12:e0188393.

187 Kipp AM, Haley C, Mukolo A. Gaps in measuring and understanding the effect of HIV stigma on health outcomes: insight from a systematic review of HIV stigma and art adherence. Ann Behav Med 2012;43:S59.

188 Mahajan AP, Sayles JN, Patel VA, et al. Stigma in the HIV/AIDS epidemic: a review of the literature and recommendations for the way forward. AIDS 2008;22 Suppl 2:S67-79.

189 McAteer Cl, Truong N-AT, Aluoch J, et al. A systematic review of measures of HIV/AIDS stigma in paediatric HIV-infected and HIVaffected populations. J Int AIDS Soc 2016;19:21204.

190 Dunbar W, Labat A, Raccurt C, et al. A realist systematic review of stigma reduction interventions for HIV prevention and care continuum outcomes among men who have sex with men. Int $J$ STD AIDS 2020;31:712-23.

191 Glick JL, Russo R, Jivapong B, et al. The PreP care continuum among cisgender women who sell sex and/or use drugs globally: a systematic review. AIDS Behav 2020;24:1312-33.
192 Liu X, Erasmus V, Wu Q, et al. Behavioral and psychosocial interventions for HIV prevention in floating populations in China over the past decade: a systematic literature review and meta-analysis. PLoS One 2014;9:e101006.

193 Poteat T, Wirtz AL, Radix A, et al. Hiv risk and preventive interventions in transgender women sex workers. Lancet 2015;385:274-86.

194 Chen D, Luo G, Meng X, et al. Efficacy of HIV interventions among factory workers in low- and middle-income countries: a systematic review. BMC Public Health 2020;20:1310.

195 Hartog K, Hubbard CD, Krouwer AF, et al. Stigma reduction interventions for children and adolescents in low- and middleincome countries: systematic review of intervention strategies. Soc Sci Med 2020;246:112749.

196 Kemp CG, Jarrett BA, Kwon C-S, et al. Implementation science and stigma reduction interventions in low- and middle-income countries: a systematic review. BMC Med 2019;17:6.

197 Lofgren SM, Nakasujja N, Boulware DR. Systematic review of interventions for depression for people living with HIV in Africa. AIDS Behav 2018;22:1-8.

198 Ma PHX, Chan ZCY, Loke AY. Self-Stigma reduction interventions for people living with HIV/AIDS and their families: a systematic review. AIDS Behav 2019;23:707-41.

199 Pantelic M, Sprague L, Stangl AL. It's not "all in your head": critical knowledge gaps on internalized HIV stigma and a call for integrating social and structural conceptualizations. BMC Infect Dis 2019;19:210.

200 Joseph Davey D, West S, Umutoni V, et al. A systematic review of the current status of safer conception strategies for HIV affected heterosexual couples in sub-Saharan Africa. AIDS Behav 2018;22:2916-46.

201 Taggart T, Grewe ME, Conserve DF, et al. Social media and HIV: a systematic review of uses of social media in HIV communication. $J$ Med Internet Res 2015;17:e248.

202 International Center for Research on Women (ICRW), STRIVE. A global HIV stigma reduction framework adapted and implemented in five settings in India: summary report, 2013. Available: https:// www.icrw.org/publications/a-global-hiv-stigma-reductionframework-adapted-and-implemented-in-five-settings-in-india/

203 Stangl A, Brady L, Fritz K. Strive technical brief: measuring HIV stigma and discrimination. Washington, D.C: International Center for Research on Women, 2018: 1-7.

204 UNAIDS. Key populations. Available: https://www.unaids.org/en/ topic/key-populations [Accessed 12 Jan 2021].

205 Global Network Of People Living with HIV (GNP+). The people living with HIV stigma index. Available: https://www.stigmaindex.org/ [Accessed February 22, 2021].

206 Schneberk T, Bolshakova M, Sloan K. Quality indicators for high need patients: systematic review protocol. prospero registration: CRD42020215917, 2020. Available: https://www.crd.york.ac.uk/ prospero/display_record.php?ID=CRD42020215917

207 De Roo ML, Leemans K, Claessen SJJ, et al. Quality indicators for palliative care: update of a systematic review. J Pain Symptom Manage 2013;46:556-72.

208 Mokkink LB, Terwee CB, Patrick DL, et al. The COSMIN checklist for assessing the methodological quality of studies on measurement properties of health status measurement instruments: an international Delphi study. Qual Life Res 2010;19:539-49.

209 Sterne JA, Hernán MA, Reeves BC, et al. ROBINS-I: a tool for assessing risk of bias in non-randomised studies of interventions. BMJ 2016;355:i4919.

210 Cochrane Methods Bias. Rob 2: a revised Cochrane risk-of-bias tool for randomized trials. Available: https://methods.cochrane.org/ bias/resources/rob-2-revised-cochrane-risk-bias-tool-randomizedtrials [Accessed 12 Jan 2020].

211 Röver C, Knapp G, Friede T. Hartung-knapp-sidik-jonkman approach and its modification for random-effects meta-analysis with few studies. BMC Med Res Methodol 2015;15:99.

212 Thomas J, O'Mara-Eves A, Brunton G. Using qualitative comparative analysis (QCA) in systematic reviews of complex interventions: a worked example. Syst Rev 2014;3:67.

213 GRADE working group. Grade. Available: https://www. gradeworkinggroup.org/ [Accessed 12 Jan 2020]. 